Avicenna Journal of Nursing and Midwifery Care - ISSN 2676-5748

\title{
Effect of Spiritual Intelligence on Quality of Nursing Care of Educational and Treatment Hospitals in Hamadan
}

\author{
Zohre Sabzianpur ${ }^{1}$, Soraya Nuri ${ }^{2 *}$
}

1. PhD., Department of Educational Technology, Faculty of Humanities, Kermanshah Branch, Islamic Azad University, Kermanshah, Iran

2. MSc., Department of Educational Technology, Faculty of Humanities, Kermanshah Branch, Islamic Azad University, Kermanshah, Iran

\begin{tabular}{lr}
\hline \multicolumn{2}{c}{ Article Info } \\
\hline & \\
Received: & $2018 / 09 / 04$ \\
Accepted: & $2018 / 10 / 10$ \\
Published Online: & $2019 / 03 / 22$
\end{tabular}

DOI: $10.30699 /$ ajnmc.27.1.18

Original Article

Use your device to scan and read the article online

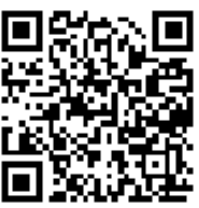

\section{Abstract}

Introduction: Spirituality and spiritual intelligence can be effective in all spheres of human life such as the spheres of work and social activities of human beings. The present research was conducted to investigate the effect of spiritual intelligence on the quality of nursing care in Hamadan University of Medical Sciences hospitals.

Methods: This descriptive-correlational study was conducted on 174 nurses working in teaching hospitals in Hamadan city, using stratified random sampling method in 2018. The data gathering tool was a questionnaire of Spiritual Quran Inventory and Quality of Nursing Care Quality Questionnaire (QUALPAC). Data were analyzed using Cronbach's alpha, Kolmogorov-Smirnov tests and Pearson correlation in SPSS21 software and structural equation modeling in PLS software.

Results: The mean age of the majority of participants was 31-40. The mean and standard deviation of spiritual intelligence dimensions included: transcendental consciousness (25.49 \pm 6.066$)$, personal meaning (18.28 \pm 4.516$)$, and critical thinking (25.22 \pm 6.152$)$ development of consciousness $(18.01 \pm 4.707)$ and in dimensions of nursing care quality, psychosocial dimension $(83.11 \pm 15.7)$, communication dimension $(40.03 \pm 7.41)$, physical dimension $(75.67 \pm 14.5)$. Transcendental consciousness, creation of personal meaning, existential thinking and development of consciousness had no significant effect on the quality of nursing care $(P>0.05)$. There was no significant relationship between spiritual intelligence and psychosocial dimension and communication quality of nursing care $(P>0.05)$. But Spiritual intelligence had a direct and significant relationship with the physical dimension of nursing care $(P<0.05)$.

Conclusion: The results of this study showed that the effect of the four components of spiritual intelligence (existential critical thinking, development of the position of consciousness, the creation of personal meaning, transcendental consciousness) on the quality of nursing care and its two dimensions, including psychosocial aspect was not significant. Only a weak relationship was found between the physical dimension of nursing care with spiritual intelligence. Finally, there is a weak correlation between spiritual intelligence and quality of nursing care.

Keywords: Intelligence, Spiritual Intelligence, Quality of Care, Nurse 


\section{بررسى تأثير هوش معنوى بر كيفيت مراقبت يرستارى بيمارستانهاى آموزشى و درمانى شهرستان همدان زهره سبزيانيور'، ثريا نورى}

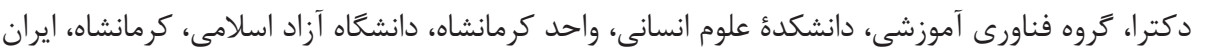

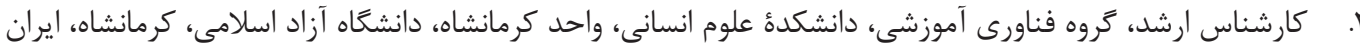

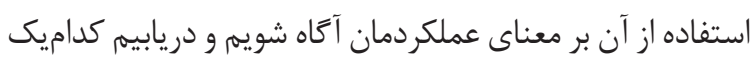

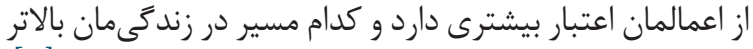

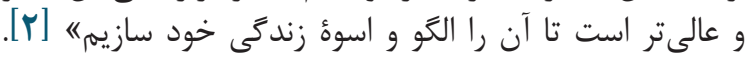

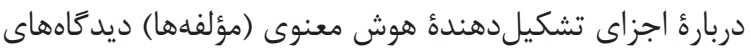
كوناگونى ارائه شده است تُكيل هوش معنوى توانايى براى رفتاركردن همراه با دلسوزى و

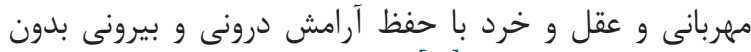

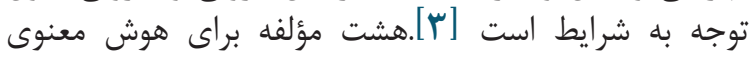

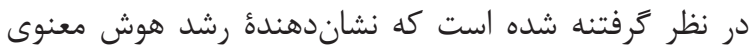

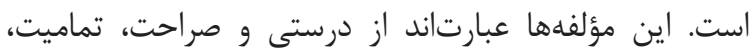

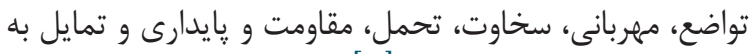

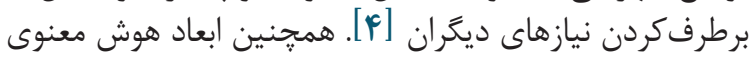

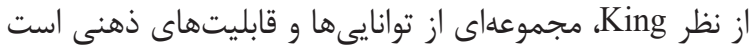

اطلاعات مقاله

تاريخ وصول: تاريخ يذيرش:

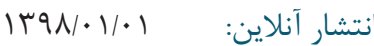

$$
\text { ثويسندهُ مسئول: }
$$

كارشناس ارشد، گَروه فناورى

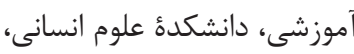

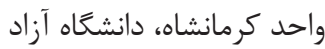
اسلامى، كرمانشاه، ايران

يست الكترونيك: s.nouri35@yahoo.com
هوش از جمله هوش معنوى بيانگر مجموعه مهارتها

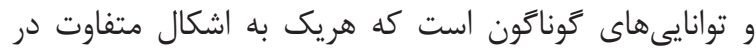

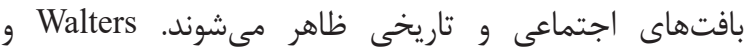
Gandner

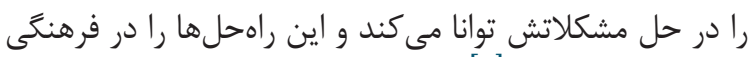

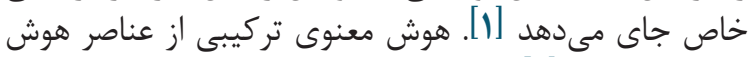
و معنويت است [1] [1]. است دang در تعريفى بيان مى كند "اهوش معنوى ظرفيت انسان

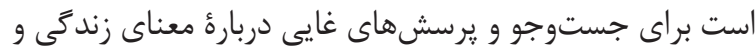

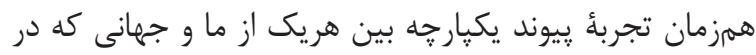

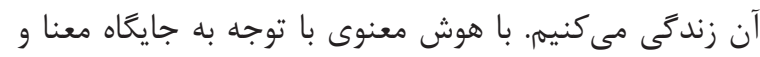

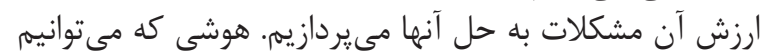

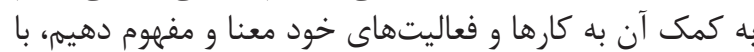


يرستار شاغل در بيمارستانهاى آموزشى-درمانى شهرستان

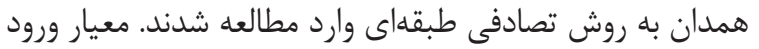

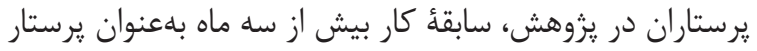

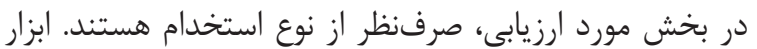

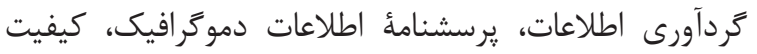

مراقبت يرستارى و هوش معنوى بود.

\section{يرسشنامة هوش معنوى}

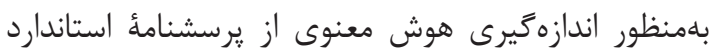

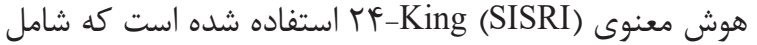

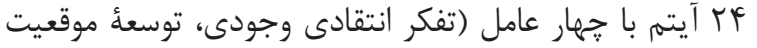

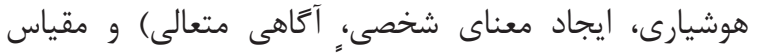

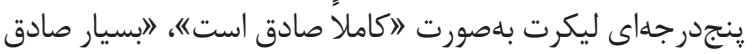

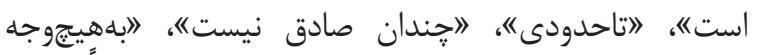

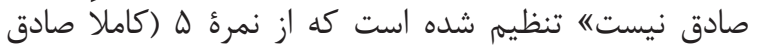

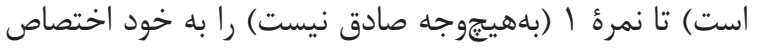

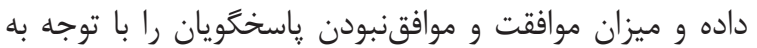

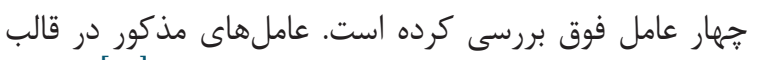

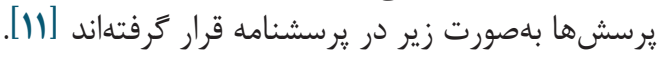

\section{يرسشنامة كيفيت مراقبت يرستارى}

بامنظور جمعآورى دادهاى مربوط به مراقبت و كيفيت Qرستارى از يرسشنامة (Quality Patient Care)

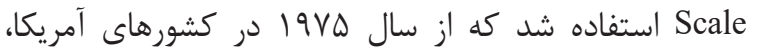

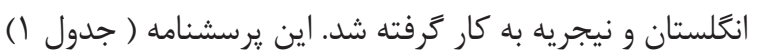

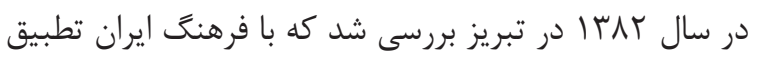

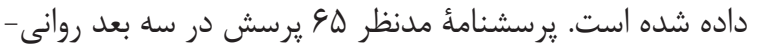

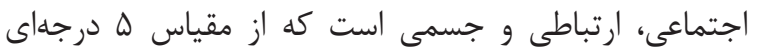

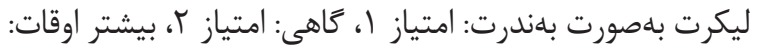

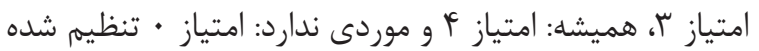

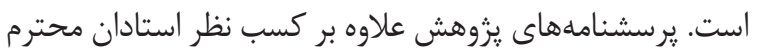

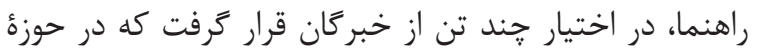

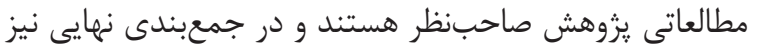

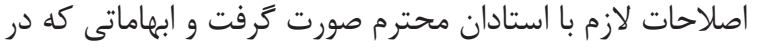

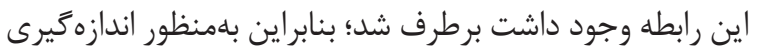

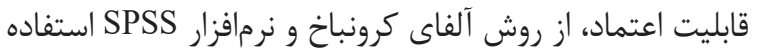

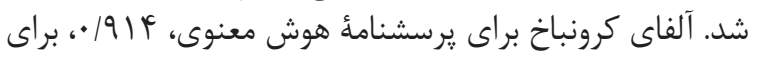

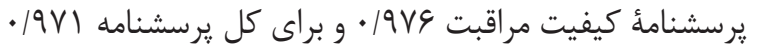

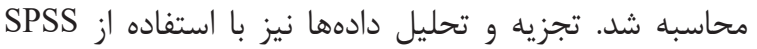
نسخه آ صورت كرفت.

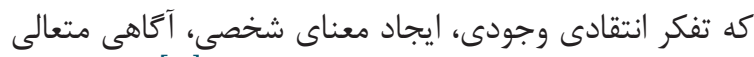

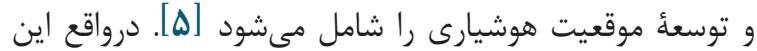

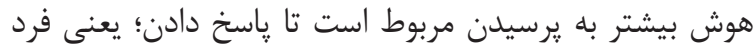

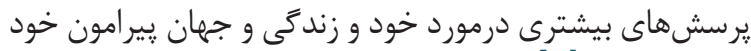
مطرح مى كند [عائ. بحث هوش معنوى نهتنها در حوزههاى فردىى، بلكه در

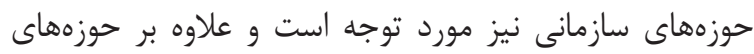

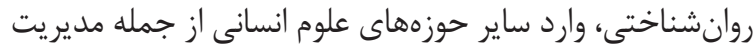

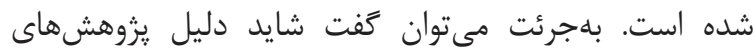

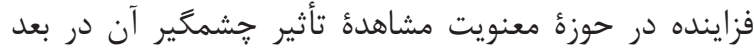

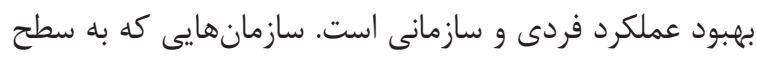

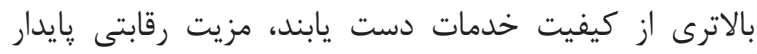

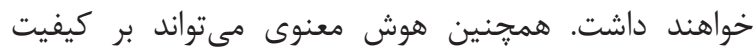

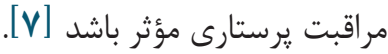

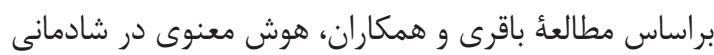

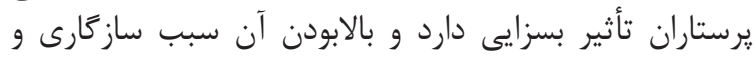

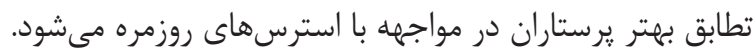
Amram

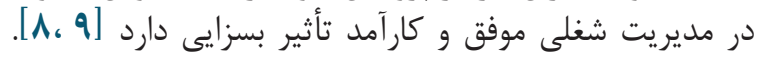

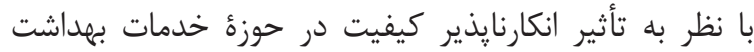

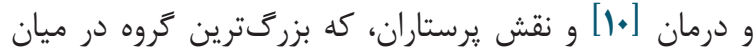

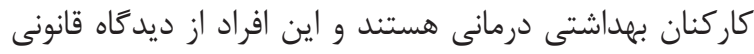

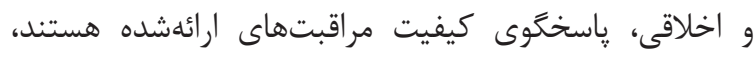

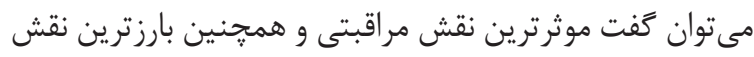

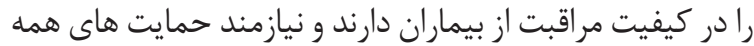

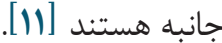

ارائُٔ مراقبتها و خدمات با كيفيت مناسب اولويتى در نظام

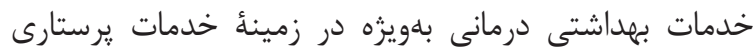

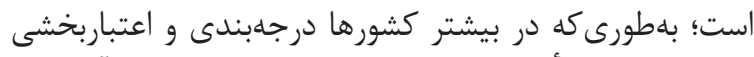

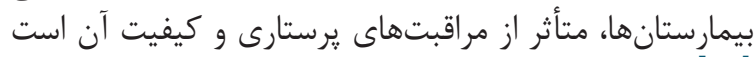

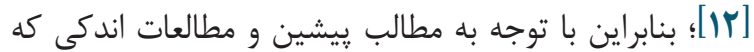

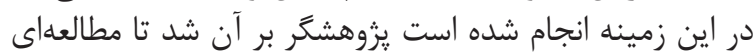

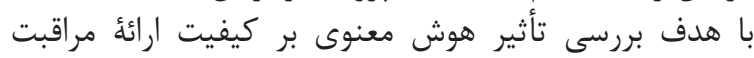

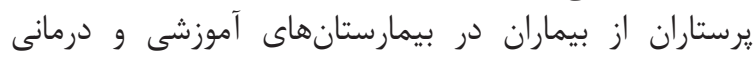

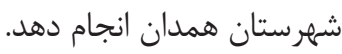

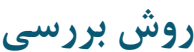

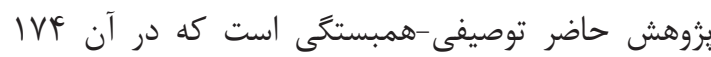

جدول ا. يرسش هاى استفادهشده براى سنجش متغيرهاى هوش معنوى شمارة برسش در برسشنامه

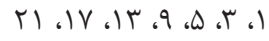

tt.

TF.19.1T، A. F

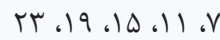

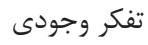

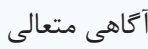

بسط حالت هوشيارى

توليد معناى شخصى
1

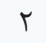

r r 
شكل I ميزان تأثير متغيرهاى مستقل بر متغير وابسته

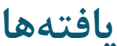

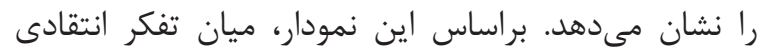

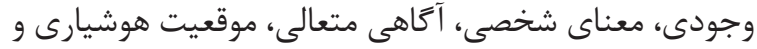

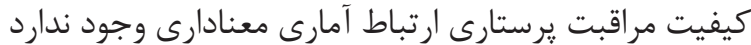

. $(P<\cdot / \cdot \Delta)$

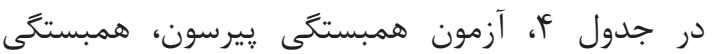

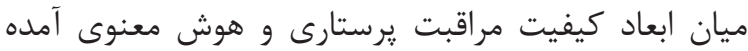

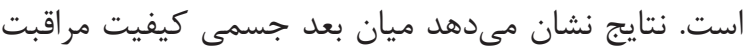

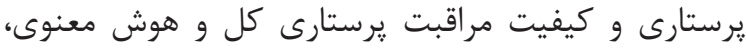

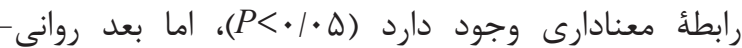

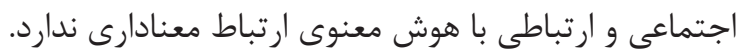

جدول r. ويرَّى هاى فردى افراد يُوهش

\begin{tabular}{|c|c|c|c|}
\hline درصد & تعداد & \multicolumn{2}{|c|}{ متغير } \\
\hline rI/A & rᄉ & مذكر & \multirow{2}{*}{ جنسيت } \\
\hline$V N / T$ & gre & مؤنث & \\
\hline$f \mid / F$ & VT & مجرد & \multirow{2}{*}{ وضعيت تأهل } \\
\hline$\Delta N / \varphi$ & $1 \cdot r$ & مؤنث & \\
\hline rq/A & $g F$ & $r \cdot-r$. & \multirow{4}{*}{ سن } \\
\hline$F+/ l$ & $v \Delta$ & rI-r. & \\
\hline $19 / 0$ & re & $|c|-\Delta$. & \\
\hline .19 & 1 & $\Delta 1-\varepsilon$. & \\
\hline $9 / \pi$ & 11 & كاردانى & \multirow{3}{*}{ ميزان تحصيلات } \\
\hline $1 \cdot 10$ & If. & كارشناسى & \\
\hline $1 \pi / r$ & r & ارشد و دكترى & \\
\hline$r r / l$ & $v \Delta$ & رسمى & \multirow{5}{*}{ نوع استخدام } \\
\hline $11 / 0$ & $r \cdot$ & رسمى_آزمايشى & \\
\hline $19 / 1$ & $r \wedge$ & ييمانى & \\
\hline$|F /|^{\mathbb{E}}$ & $r \Delta$ & قراردادى & \\
\hline $14 / 9$ & re & طرح و تعهد & \\
\hline $\mid 1 / F$ & rt & زير ه سال & \multirow{6}{*}{ سابقه كار } \\
\hline ET/D & $V F$ & $\Delta-1$. & \\
\hline$T r / F$ & rq & $1 \cdot-10$ & \\
\hline$q / \pi$ & 11 & $\mid \Delta-r$. & \\
\hline$V / \Delta$ & r (r & $r \cdot-r Q$ & \\
\hline$r / q$ & $\Delta$ & $r \Delta-r$. & \\
\hline
\end{tabular}

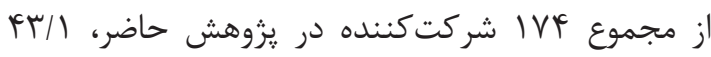

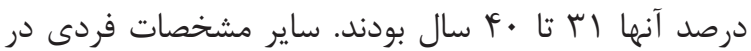

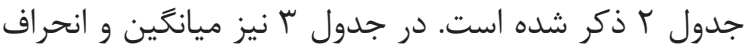

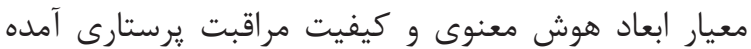

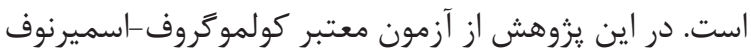

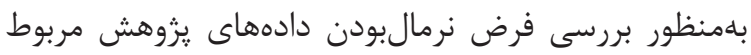

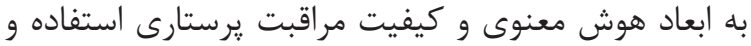
مشخص شد همأ متغيرهاى بررسى توزيع نرمالى دارند. 
جدول r. ميانغين و انحراف معيار ابعاد هوش معنوى و كيفيت مراقبت برستاران واحدهاى يثوهش

ميانتين و انحراف معيار

$|N / T \Lambda \pm F / \Delta| 9$

$r \Delta / Y T \pm 9 / \mid \Delta T$

$r \Delta / q q \pm q / . q 9$

$|N / \cdot| \pm F / V \cdot V$

$\Lambda V+Y \mid / F+1$

$V \Delta / \mathcal{G} \pm \mid F / \Delta$

$\Lambda \mu / 1) \pm 1 \Delta / V$

$f \cdot / \cdot r \pm V / f \mid$

$19 N / N 1+r V / 8 \mid$

\section{ابعاد}

ايجاد معناى شخصى

تفكر انتقادى وجودى

$$
\text { آكاهى متعالى }
$$

توسعأ موقعيت هوشيارى

$$
\text { هوش معنوى }
$$

$$
\text { بعد جسمى كيفيت مراقبت يرستارى }
$$

بعد روانى -اجتماعى كيفيت مراقبت يرستارى

بعد ارتباطى كيفيت مراقبت يرستارى

كيفيت مراقبت يرستارى

جدول F. بررسى همبستكى پِيرسون بين ابعاد كيفيت مراقبت يرستارى و هوش معنوى

\section{سطح معنادارى (P)}

\section{ضر يب همبستغى (r) ( )}

هوش معنوى

بعد بعد روانى -جتماعى كيفيت مراقبت يرستارى

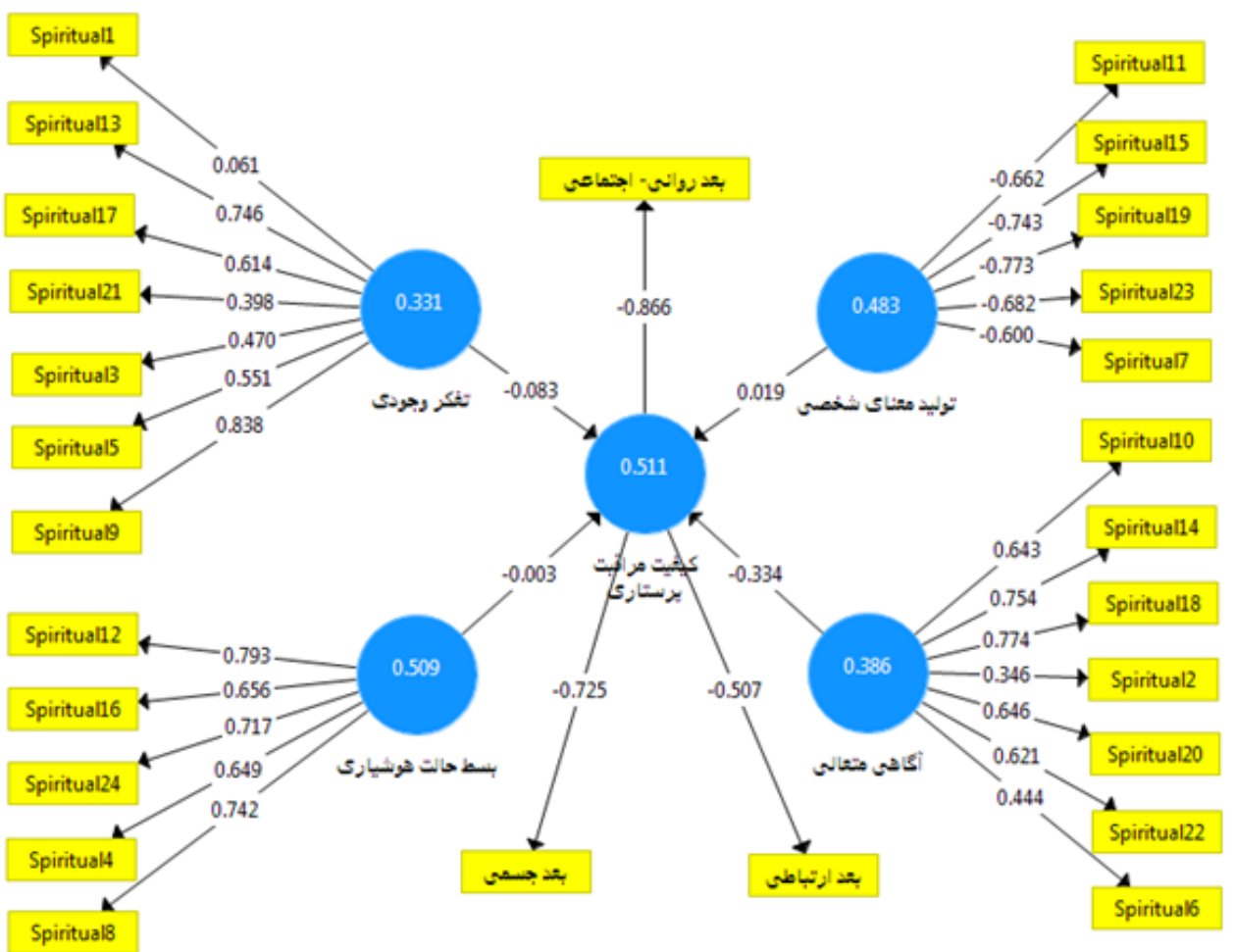

شكل ا. نتيجةُ اجراى مدل ِييشنهادى پروهش با ضرايب استاندارد 


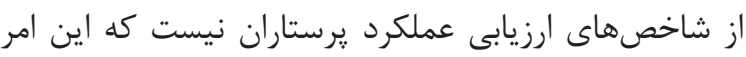

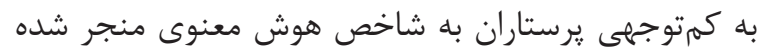

كمبود نيروى انسانى در محيطهاى آموزشى و درمانى

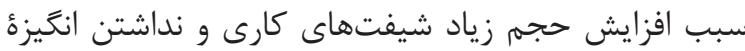

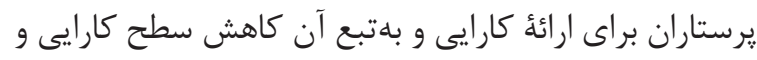

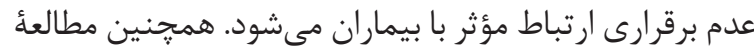
Hansen

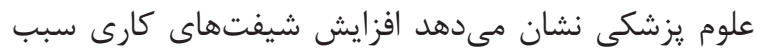

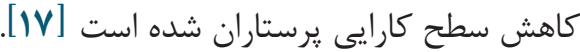

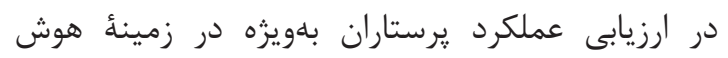

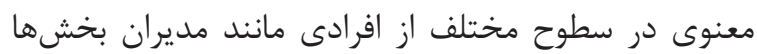

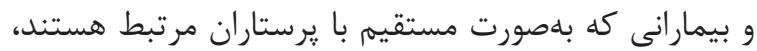

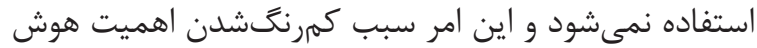

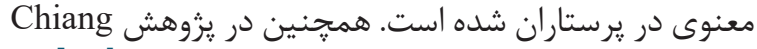

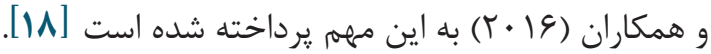

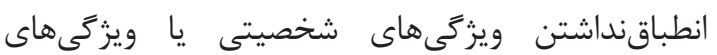

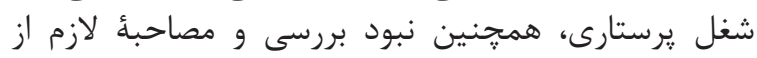

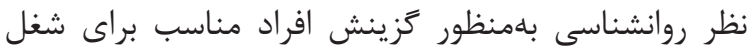

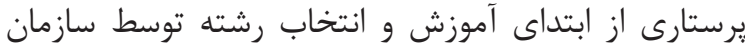

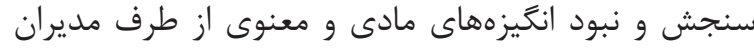

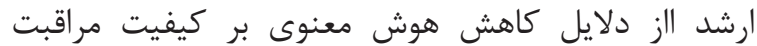

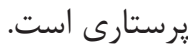

يكى ديخر از علل ارتباطنداشتن هوش معنوى و كيفيت

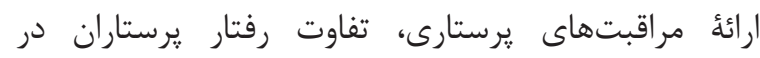

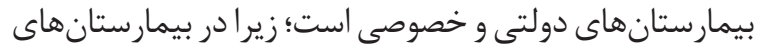

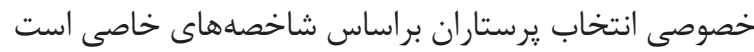

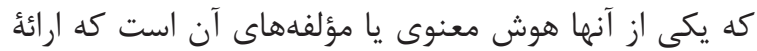

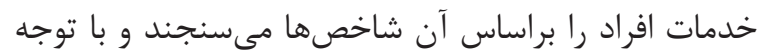

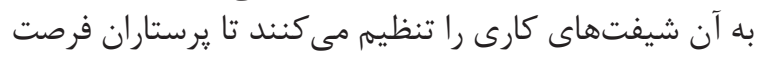

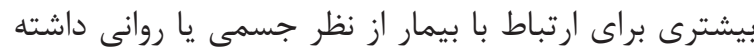

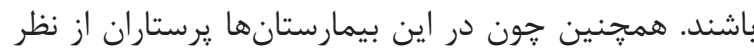

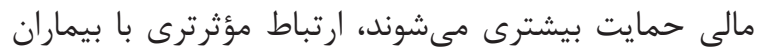

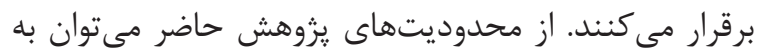

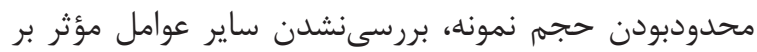

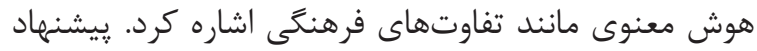

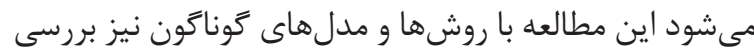

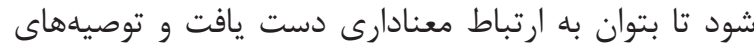

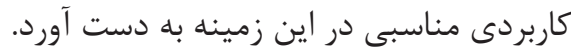

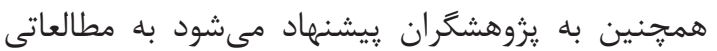

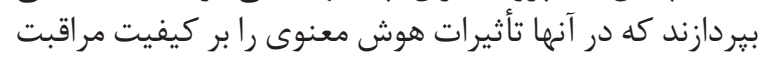

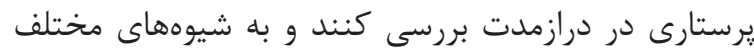
تأثير هوش معنوى بر كيفيت مراقبت برستارى بيردازئند

\section{نتيجه تيرى}

هوش معنوى با تقويت شخصيت و باورهاى يرستاران

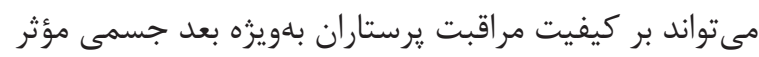

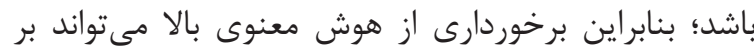

\section{بحث}

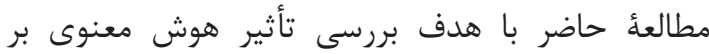

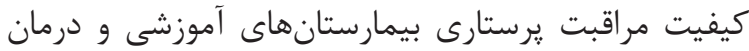

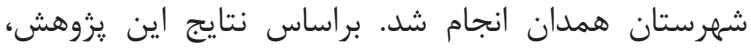

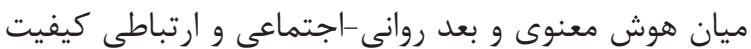

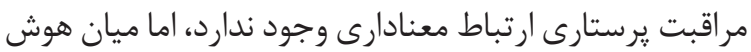

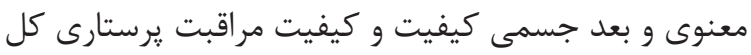

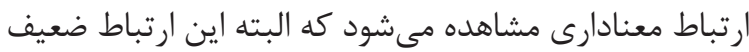

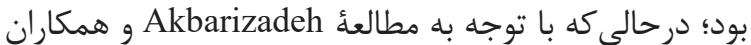

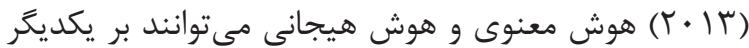

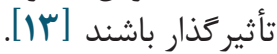

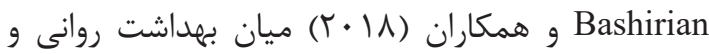

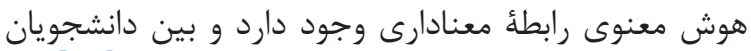

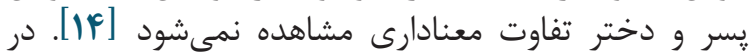

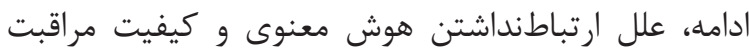

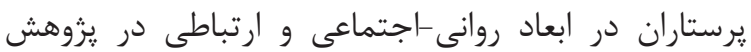

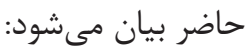

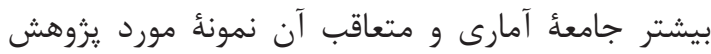

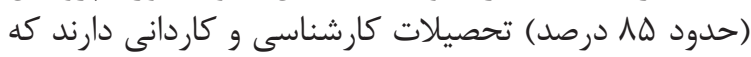

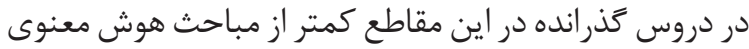

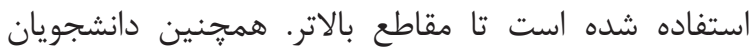

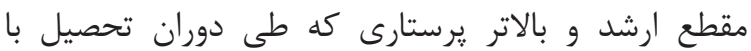

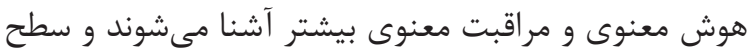

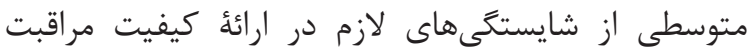

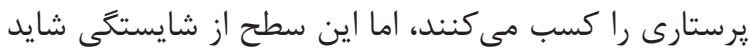

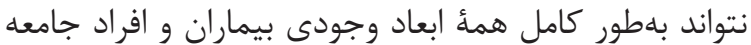

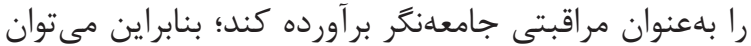

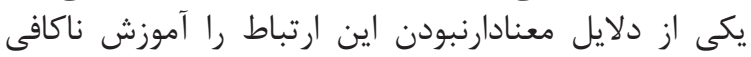

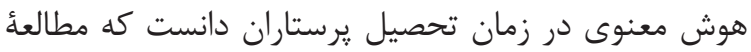
Nasehi

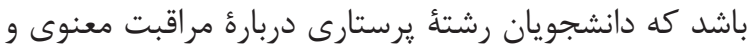

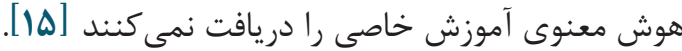

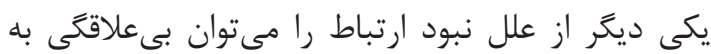

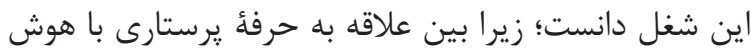

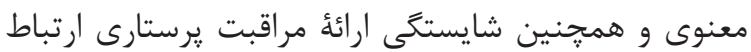

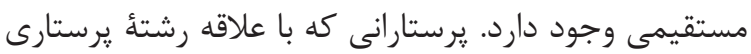

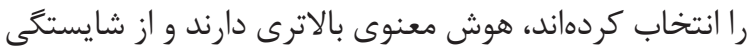

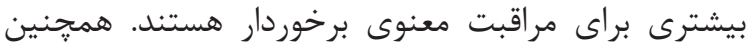

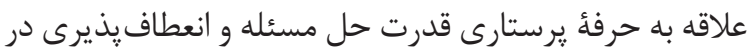

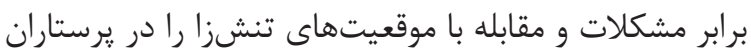

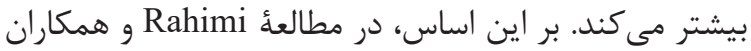

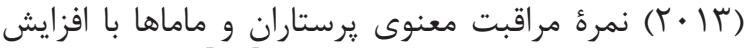

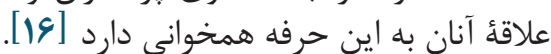

با توجه به اينكه ارزشيابى عملكرد، روشى مناسب براي تراى

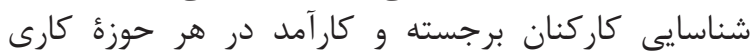

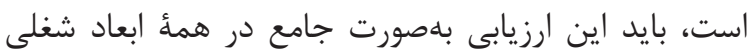
حرفهاى خاص انجام شود، اما متأسفانه شاخص هوش درئ معند ابعاد شعلى 


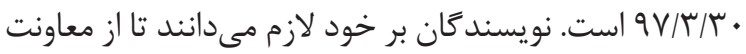

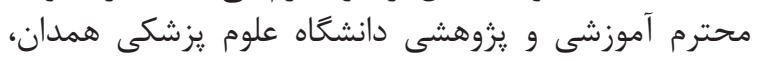

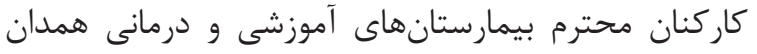

$$
\begin{aligned}
& \text { كمال تشكر و قدردانى را داشته باشندان } \\
& \text { تعارض در منافع } \\
& \text { بين نويسندكان هيجگَّنه تعارضى در منافع وجود ندارد. }
\end{aligned}
$$

$$
\begin{aligned}
& \text { TF بر YF تأثير هوش معنوى بر كيفيت مراقبت برستارى } \\
& \text { ارتقاى كيفيت كار يرستاران و كادر درمان و درنهايت سلامت }
\end{aligned}
$$

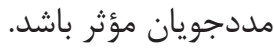

$$
\begin{aligned}
& \text { سياسگز ارى }
\end{aligned}
$$

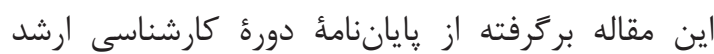

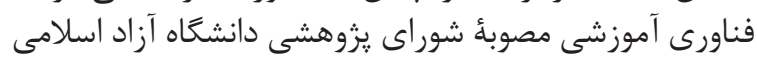

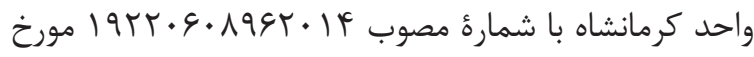

\section{References}

1. Moeini B, Barati M, Farhadian M, Ara MH. The association between social support and happiness among elderly in Iran. Korean journal of family medicine. 2018; 39(4):260.

2. Yang KP, and Mao XY. A Study of Nurses' Spiritual Intelligence: A Cross-Sectional Questionnaire Survey. International Journal of Nursing Studies. 2007; 44(6):999-1010. https://doi. org/10.1016/j.ijnurstu.2006.03.004

3. Dev RD, Kamalden TF, Geok SK, Abdullah MC, Ayub AF, Ismail IA. Emotional Intelligence, Spiritual Intelligence, SelfEfficacy and Health Behaviors: Implications for Quality Health. International Journal of Academic Research in Business and Social Sciences. 2018; 8(7):794-809.

4. Dev RD, Kamalden TF, Geok SK, Ayub AF, Ismail IA. Spiritual intelligence on health behaviours among Malaysian university students in a Malaysian public university: The mediating role of self efficacy. Malaysian Journal of Movement, Health \& Exercise. 2018; 7(2).

5. King DB, De Cicco TL. A viable model and self-report measure of spiritual. International Journal of Transpersonal Studies. 2009; 28(1):6885.

6. Liu M, Kunaiktikul W, Senaratana W, Tonmukayakul O, Eriksen L. Development of competency inventory for registered nurses in the People's Republic of China: scale development. Int J Nurs Stud 2007; 44(5):805-13.

7. Pant N, Srivastava SK. The Impact of Spiritual Intelligence, Gender and Educational Background on Mental Health Among College Students. Journal of religion and health. 2019; 58(1):87-108.

8. Bagheri F AF, Hatami H. The relationship between nurses' spiritual intelligence and happiness in Iran. Procedia Soc Behav Sci. 2010; 5(15):56-61.

9. Amram JY. The Contribution of Emotional and Spiritual Intelligences to Effective Business Leadership. Institute of Transpersonal Psychology; 2009.

10. Babamiri M, Moeini B, Tahmasian H, Barati M, Roshanai G. The Study of Sleep Health Education
Effect on Sleep Quality Among Lorestan Nursing Personnel. Iranian Journal of Ergonomics. 2017; 4(4):8-13. http://journal.iehfs.ir/article-1-341-en. $\underline{\mathrm{html}}$

11. Miri KH, Keshavarz A, SHirdelzadeh S, Parsa P. The Relationship Between Nurses'spiritual Elligence and Quality of Nursing Care Based on Nurses' and Patients' viewpoints. The Journal of Urmia Nursing and Midwifery Faculty. 2015; 13(6):518-24.

12. Lambert Va, and Lambert Ce. Nurses' Workplace Stressors and Coping Strategies. Indian Journal of Palliative Care. 2008; 14(1):38. https://doi. org/10.4103/0973-1075.41934

13. Akbarizadeh F, Jahanpour F, Hajivandi A. The relationship of general health, hardiness and spiritual intelligence relationship in Iranian nurses. Iranian journal of psychiatry. 2013; 8(4):165.

14. Bashirian S, Barati M, Mohammadi Y, Moaddabshoar L, Dogonchi M. An Application of the Protection Motivation Theory to Predict Breast Self-Examination Behavior among Female Healthcare Workers. European Journal of Breast Health. 2019; 15(2):90-97.

15. Nasehi A, Rafiei H, Jafari M, Borhani F, Sabzevari S, Baneshi M, et al. Survey of Nurse's Students Competencies for Delivering Spiritual Care to Their Patients. Journal of Clinical Nursing and Midwifery. 2013; 2(2):1-9.

16. Rahimi N, Nouhi E, and Nakhaee N. Spiritual Well-Being and Attitude Toward Spirituality and Spiritual Care in Nursing and Midwifery Students. Iran Journal of Nursing. 2013; 26(85):5565.

17. Hansen AB, Stayner L, Hansen J, Andersen ZJ. Night shift work and incidence of diabetes in the Danish Nurse Cohort. Occupational and Environmental Medicine. 2016; 73(4):262-268.

18. Chiang YC, Lee HC, Chu TL, Han CY, Hsiao YC. The impact of nurses' spiritual health on their attitudes toward spiritual care, professional commitment, and caring. Nursing Outlook. 2016; 64(3):215-224. 